\title{
Modeling of the Inking Apparatus of the Sheet Printing Machine
}

\author{
Eldar A. Aliyev
}

Department of Theory of Mechanisms and Machines, Azerbaijan Technical University, H. Javid Ave. 25. Baku, AZ1073, Azerbaijan

Corresponding Author Email: elab57@mail.ru

https://doi.org/10.18280/jesa.520602

Received: 25 July 2019

Accepted: 11 October 2019

\section{Keywords:}

inking apparatus, offset printing, distribution model, dynamic

characteristic

\begin{abstract}
The ink apparatus was considered as a dynamic system formed by a combination of rollers and cylinders. The transition characteristic of the ink apparatus was considered as a natural reaction of this model to various external influences. For research, a single-capacitance model of an ink apparatus is proposed. The flow of ink through a series of rollers and cylinders that form a baseline is considered. To determine the amount of ink, the power conditions of the ink machine are considered. Presumably, the power of the ink unit is pulsed. The amount of ink passing through each contact node in one printing cycle. A model of the distribution of ink layers in the ink apparatus of a sheet printing machine is built. A method for calculating the distribution of ink layers in the ink apparatus for letterpress and offset printing machines has been developed. The regularity of the distribution of ink across the maximum and minimum layers is established. Also determined the total amount of ink accumulated in the ink apparatus. For the unsteady mode, the change in the volume of ink in the stream was characterized by the equation of a single-capacitance model of the ink apparatus. The equation of non-stationary mode is obtained. The dynamic characteristic of a single-capacitance model made it possible to determine the unevenness of the ink layer on the form in the case of a pulsed power supply of the ink apparatus. The extension of the proposed methodology to ink apparatus with branched streams of ink makes it possible to use this method for the study of ink apparatus of different designs. Also, the presentation of an ink apparatus in the form of a single-capacitance object creates the prerequisites for a more accurate description of its properties in transition modes.
\end{abstract}

\section{INTRODUCTION}

It is known that the main purpose of the ink printing system is to provide a given thickness of the ink layer on the prints when printing different types of products. Therefore, the ink system can be considered as an object of regulation. Adjustable, that is, the initial, value is the thickness of the layer of ink on the prints, the input is the supply of ink. Changing the type of printed matter requires changing the printing form. This can be considered as a change in the nature of the disturbance and the load on the inking system.

Inking devices of offset printing machines [1] are the most important technological units, the dynamic and static properties of which significantly affect the print quality. Therefore, the urgent task is to develop mathematical methods for researching the work and further model-oriented design of inking devices based on software and hardware.

The purpose of this work is to create a scientific basis for structural and mathematical modeling of a high-pressure and high-sheet offset printing apparatus for printing machines.

To achieve this goal, the following tasks were set:

Modeling of the inking apparatus as a control object associated with the movement and ink flow of the printing process;

Determination of the nutritional condition for the development of a single-capacitive mathematical model of the inking apparatus;

Development of methods for the distribution of ink layers in an inking apparatus for high and sheet offset printing machines

\section{LITERATURE REVIEW AND PROBLEM STATEMENT}

To describe the dynamics of the inking apparatus in work [2], approximate mathematical models with pure delay were used, which are obtained by the experimental study of several prototypes of the inking apparatus or one variable prototype. The theoretical path corresponds to modeling the processes of transferring ink and a moisturizing solution in the inking apparatus by describing physical processes with the subsequent use of software implemented on a computer. However, the model of the inking apparatus is not described in the work.

Using a high-precision force measuring device manufactured in the laboratory [3], a method for measuring the adhesion force when the ink film is torn from a blanket is developed. Using the proposed method, the adhesion properties of industrial inks of Ag nanoparticles, varying the film thickness and solids content, were evaluated. However, in this work, individual ink-transporting units of the ink apparatus during ink transfer were not considered.

To improve the quality of color offset printing and to learn the process of ink transfer, the article [4] uses the method of theoretical analysis and experiment. The speed and pressure of 
the ink flow field are analyzed by deformation of the rubber roller and rotation of the two rollers. The effective ink layer thickness is removed when the ink transfer rate can be obtained. The authors obtained the deformation of the rubber roller and the ink transfer rate under various conditions, and analyzed factors such as rotational speed, deformation of the rubber roller, the thickness of the ink layer between the two rollers, which affect the ink flow and transfer speed.

Verkhola et al. [5] developed mathematical models of the elements of inking devices and based on them a model of the ink-printing system of a printing offset machine from Heidelberg GTO 52 was compiled. This mathematical model describes the process of rolling and transferring ink from an ink-spraying device to prints. In addition, the model makes it possible to take into account the different modes of operation of the spray device. Based on the mathematical model, an information technology for research and analysis of the nature of the distribution of ink and its effect on the thickness of the layer, which is transmitted to the output of the ink-printing system, was created. However, the proposed model is intended for small-format machines with a simple inking device.

A number of scientists have revealed various approaches to the mathematical description of inking devices. Thus, the calculation was based on a system of algebraic equations that reflects the addition and division of ink layers in contact zones [6-8]. The use of these equations makes it possible to solve the static problem of the distribution of ink along the inking apparatus, as well as the dynamic problem of transferring layers of ink to the form and print; moreover, to solve the dynamic problem, it is proposed to consider systems of algebraic equations composed for a series of sequential cycles. Considering the duration of establishing a stationary mode, ranging from several tens to several hundreds of cycles, one can judge the great complexity and complexity of the computational work associated with this method.

A model of an inking device was proposed by Alekseyev [9], based on the consideration of the operations of splitting individual volumes of ink into many discrete strips and forming a continuous non-uniform layer from them by summing. The mathematical model of the inking apparatus is represented in matrix form, and its transformation is carried out by methods of vector algebra. The complexity of these calculations is such that the use of computer technology is also required. However, in this work this path is not investigated and structural modeling of the inking apparatus is not considered.

Ink transfer to a printing form was studied taking into account the surface roughness of the printing form [10]. However, the paper does not consider structural modeling of the inking apparatus.

The Reynolds equation for ink transfer was derived on the basis of the theory of elastohydro dynamic lubrication, and a model of the relationship between the roller parameters, speed, pressure and ink thickness in the gap of the rollers was established using the Hertz contact theory [11, 12]. The influence of the speed, pressure and size of the roller on the thickness of the ink in the gaps of the rollers was analyzed. In addition, the ink transfer model was modified subject to the retention of ink in the roller gaps. The effect of print speed on ink performance was analyzed using computer simulation. However, in these works, structural modeling of the inking apparatus was not considered.
The discrepancy between the various structures and parameters of the inking system of modern offset printing was investigated [13]. The surfaces of the inking rollers and cylinders were discretized using computer simulation, on the basis of which a mathematical model of constant time was created in a system of periodic and continuous ink supply. And then the feed system and the vibration system are analyzed. The simulation result shows that the ink supply system, the method of vibration of the vibrator, the coating of the plate has a great influence on the dynamic properties. However, in this work, structural modeling of the inking apparatus is also not considered in studying the influence of the inking supply system.

Based on a non-oriented graph [14], a system for manual input of offset printing was considered as a complex nonoriented graph and a network diagram was created. On this basis, in accordance with the distribution of the surface of the ink roller and the creation of a dynamic two-dimensional array for recording changes in the thickness of the ink on the ink roller, the process of ink transfer during printing was really and intuitively reproduced. However, in this work, the structural modeling of the inking apparatus was also not investigated.

Based on the analysis of published scientific papers, the following conclusion can be made about the state of the problem of mathematical modeling by the printing process.

The development of methods for the mathematical description of the printing process, in relation to sheet offset printing, goes in several directions.

One of which is a mathematical study of the movement of ink and a moisturizing solution in the ink and moisturizer and their interaction on a printed form in order to determine the conditions for obtaining high-quality prints.

Study of the movement and interaction of the main material flows during sheet offset printing by traditional methods of mathematical analysis and theoretical mechanics is not able to cover complex and diverse dynamic phenomena and cannot give a complete mathematical description of the printing process as an automatic object management.

\section{SINGLE-CAPACITANCE MODEL OF THE INKING APPARATUS}

Suppose that the movement of ink on the surface of a roller or cylinder is characterized by a flow $Q$ through a crosssectional area $\mathrm{S}=l_{j} h$.

$$
Q=\iint_{S} V_{n} d S
$$

Under these conditions, we take the printing speed constant $v_{n}=$ const and limit the flow to the width of the elementary zone $l_{j}=$ const . Then we find that the flow of ink is uniquely determined by the thickness of the ink layer

Consider the flow of ink through a sequential chain of rollers and cylinders $1,2, \ldots, k, k+1, k+2$, which form a baseline (Figure 1a). We accept the following modeling conditions: when leaving any node $\mathrm{i}$ in the direction of direct flow, the contact layer $h_{i}$ is multiplied by the transmission coefficient $\beta_{i}$, and in the direction of the return flow, by the coefficient $\left(1-\beta_{i}\right)$ (Figure 1b). The ratio of the formed layers is characterized by a splitting coefficient. 


$$
B_{i}=\left.\frac{\beta_{i}}{1-\beta_{i}}\right|_{\beta_{i=0,5}}=1
$$

When transferring ink from the knurling roller $(\mathrm{k})$ to the plate cylinder $(\mathrm{k}+1)$ with a fill factor $\sigma$ (provided that the relief is level).

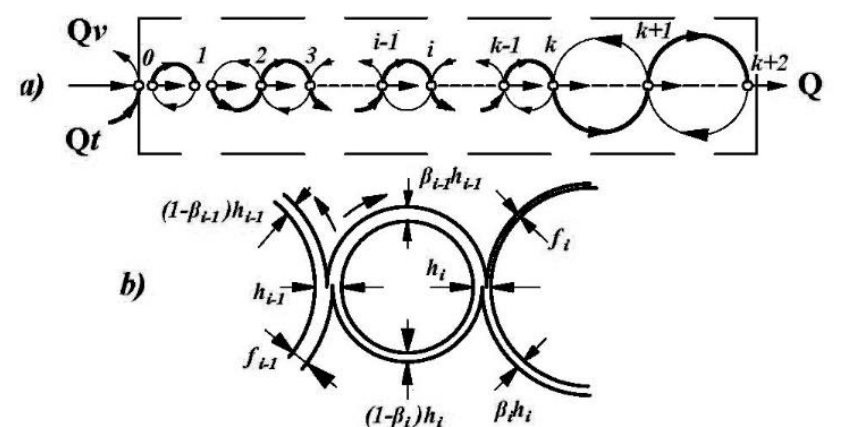

c)
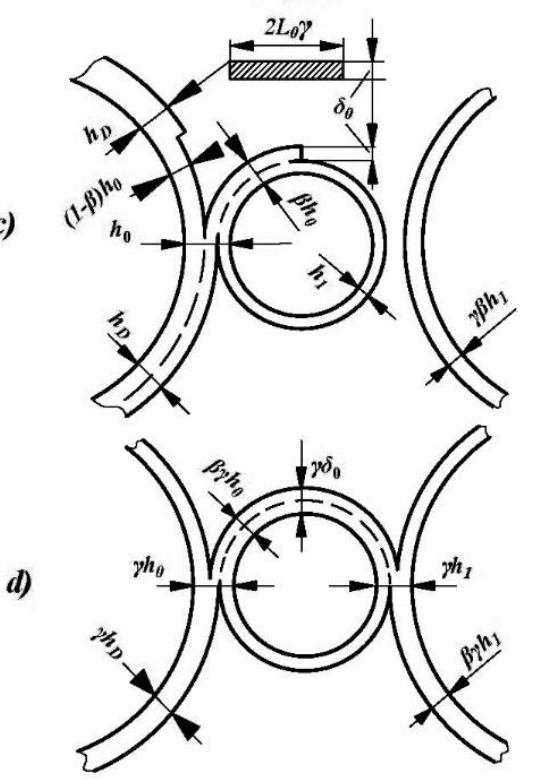

Figure 1. Ink flow model: a- baseline; b- ink transfer through the roller; c- pulse power mode; $d$ - continuous power

$$
B_{k}=\left.\frac{\sigma \beta_{k}}{1-\sigma \beta_{k}}\right|_{\beta_{k=0,5}}=\frac{\sigma}{2-\sigma}
$$

When transferring ink from a mold to rubber and from rubber to paper

$$
B_{k+1}=\frac{\beta_{k+1}}{1-\beta_{k+1}} ; \quad B_{k+2}=\frac{\beta_{k+2}}{1-\beta_{k+2}}
$$

in letterpress- $B_{k+1}=B_{p}=\frac{\beta_{p}}{1-\beta_{p}}$

in offset printing- $B_{k+2}=B_{p}=\frac{\beta_{p}}{1-\beta_{p}}$

To determine the amount of ink supplied to the inking apparatus in the average for one printing cycle and the amount of ink transferred to the print, it is necessary to consider the power conditions of the inking apparatus.

\subsection{Determining the power condition of the inking apparatus}

Suppose that the power of the ink unit is pulsed by the oscillating transfer roller: $\mathrm{T}=m \tau_{0}$ - the power cycle, where - $m$ is the number of printing cycles $\tau_{0} ; \gamma T$ power pulse duration the time it takes for the duct cylinder to rotate a certain angle; $\delta_{0}$ - pulse amplitude - the thickness of the supplied portion of ink, which is determined by the equation (Figure 1c).

$$
\delta_{0}=h_{D}-(1-\beta) h_{0}
$$

The amount of ink supplied on average per print cycle is

$$
\begin{aligned}
& g_{0}=\frac{1}{m} \gamma \delta_{0}=\gamma_{0} \delta_{0}=\gamma_{0} h_{D}-(1-\beta) \gamma_{0} h_{0}= \\
& =\beta \gamma_{0} h_{0}-(1-\beta) \gamma_{1},
\end{aligned}
$$

Whence it follows that pulse power with an amplitude $\delta_{0}$, period $\mathrm{T}$ and duty cycle $\gamma$ is equivalent to pulse power with a period $\tau_{0}$ and duty cycle $\gamma_{0}=\gamma / m$ or continuous power supply with a thickness of the supply layer $\gamma_{0} \delta_{0}$ (Figure 1d).

Assuming that the amount of ink entering node $i$ is equal to the amount of ink being drawn from the node, we can write (Figure 1b).

$$
g_{i}=\beta_{i-1} h_{i-1}-\left(1-\beta_{i}\right) h_{i}=\beta_{i} h_{i}-\left(1-\beta_{i+i}\right) h_{i+i}
$$

In stationary mode, the amount of ink $g_{i}$ passing through each contact node for one printing cycle is constant and equal to the amount of ink supplied to the ink supply $g_{0}$ unit on average for one printing cycle and the amount of ink transferred to the print:

$$
\begin{aligned}
& g_{0}=\delta_{0} \gamma_{0}=\ldots=\beta_{i-1} h_{i-1}-\left(1-\beta_{i}\right) h_{i}= \\
& =\beta_{i} h_{i}-\left(1-\beta_{i+i}\right) h_{i+i}=\ldots=\sigma h
\end{aligned}
$$

where, $h$ is the thickness of the ink layer on the print.

3.2 The study of modeling the inking apparatus and the method of calculating the distribution of layers of ink the inking apparatus

Based on Eq. (8) taking into account the splitting coefficients $B_{\mathrm{i}}$ build a model of the distribution of ink layers:

$$
\begin{aligned}
& \frac{\beta_{i} h_{i}}{h}=\frac{\left[1-\left(1-\beta_{i}\right)\right] h_{i}}{h}= \\
& =\sigma\left[1+\frac{1}{B_{i+1}}\left(1+\frac{1}{B_{i+2}}\left(1+\ldots .+\frac{1}{B_{k}}\left(1+\frac{1}{B_{k+1}}\left(1+\frac{1}{B_{k+2}}\right) \ldots\right)\right)\right)\right]_{(9)}
\end{aligned}
$$

At $\beta_{i}=0,5(i=1,2, \ldots k)$ formula (9) gets a simple form

$$
\frac{\beta_{i} h_{i}}{h}=\sigma(k-i)+\frac{c_{0}}{2}(2-\sigma)
$$

where, $\quad C_{0}=2 / \beta_{p}$, for letterpress machines $\left(\beta_{k+1}=\beta_{p}\right.$; $\left.1+\frac{1}{\beta_{k+2}} \equiv 1\right)$ 
$C_{0}=2 \frac{2+\beta_{p}}{1-\beta_{p}}$-for offset printing machines $\left(\beta_{k+1}=0,5\right.$; $\left.\beta_{k+2}=\beta_{p}\right)$

Ink distribution in inking devices under conditions

$$
\begin{gathered}
h_{i}=\beta_{i} h_{i}+\left(1-\beta_{i}\right) h_{i} ;\left(1-\beta_{i}\right) h_{i}=\frac{1}{B_{i}} \beta_{i} h_{i} ; \\
h_{i}^{a v e}=\frac{1}{2 \pi}\left[\alpha_{i} \beta_{i-1} h_{i-1}+\left(1-\alpha_{i}\right)\left(1-\beta_{i}\right) h_{i}\right] \\
\frac{\beta_{i} h_{i}}{g_{0}}=1+\frac{1}{B_{i+1}}\left(1+\frac{1}{B_{i+2}}\left(1+\ldots .+\frac{1}{B_{k}}\left(1+\frac{1}{B_{k+1}}\left(1+\frac{1}{B_{k+2}}\right) \ldots\right)\right)\right)
\end{gathered}
$$

as well as

$$
\begin{gathered}
B_{0}=\ldots=B_{i}=\ldots=B_{k-1}=\frac{\beta}{1-\beta}=1 \quad B_{k}=\frac{\sigma}{2-\sigma} ; \\
B_{k+1}=\frac{\beta_{k+1}}{1-\beta_{k+1}} ; \quad B_{k+2}=\frac{\beta_{k+2}}{1-\beta_{k+2}}
\end{gathered}
$$

where, $\alpha_{i}$ - the displacement coefficient, which characterizes part of the ink path along the periphery of the roller between the contact nodes when the flow is moving in the forward direction; define in the following form:

For letterpress machines:

$$
\begin{gathered}
\frac{\beta_{k+1} h_{k+1}}{g_{0}}=1 \\
\frac{\sigma \beta h_{k}}{g_{0}}=1+\frac{1}{B_{k+1}} \\
\frac{\beta h_{k-1}}{g_{0}}=1+\frac{1}{B_{k}}\left(1+\frac{1}{B_{k+1}}\right) \\
\frac{\beta h_{i}}{g_{0}}=k-i+\frac{1}{B_{k}}\left(1+\frac{1}{B_{k+1}}\right) \\
\frac{\beta h_{1}}{g_{0}}=k-i+\frac{1}{B_{k}}\left(1+\frac{1}{B_{k+1}}\right) \\
\frac{\beta h_{0}}{g_{0}}=k+\frac{1}{B_{k}}\left(1+\frac{1}{B_{k+1}}\right) \\
\frac{\gamma h_{D}}{g_{0}}=k+1+\frac{1}{B_{k}}\left(1+\frac{1}{B_{k+1}}\right)
\end{gathered}
$$

Also for offset printing machines:

$$
\begin{gathered}
\frac{\beta_{k+2} h_{k+2}}{g_{0}}=1 \\
\frac{\beta_{k+1} h_{k+1}}{g_{0}}=1+\frac{1}{B_{k+2}} \\
\frac{\sigma \beta h_{k}}{g_{0}}=1+\frac{1}{B_{k+1}}\left(1+\frac{1}{B_{k+2}}\right)
\end{gathered}
$$

$$
\begin{aligned}
& \frac{\beta h_{k-1}}{g_{0}}=1+\frac{1}{B_{k}}\left(1+\frac{1}{B_{k+1}}\left(1+\frac{1}{B_{k+2}}\right)\right) \\
& \frac{\beta h_{i}}{g_{0}}=k-i+\frac{1}{B_{k}}\left(1+\frac{1}{B_{k+1}}\left(1+\frac{1}{B_{k+2}}\right)\right) \\
& \frac{\beta h_{0}}{g_{0}}=k+\frac{1}{B_{k}}\left(1+\frac{1}{B_{k+1}}\left(1+\frac{1}{B_{k+2}}\right)\right) \\
& \frac{\gamma h_{D}}{g_{0}}=k+1+\frac{1}{B_{k}}\left(1+\frac{1}{B_{k+1}}\left(1+\frac{1}{B_{k+2}}\right)\right)
\end{aligned}
$$

Based on the data obtained, we construct an ink flow distribution diagram for the baseline (Figure 2). With $\sigma=0$ on all rollers $i \leq k$ a constant layer is established $C_{0}$; at $i>k$ the layer is equal to zero (there is no transfer of ink to the form, since $\sigma=0$ ). With $\sigma=1$, the distribution diagram is a descending step line with step I. At $\sigma<1$, the step decreases proportionally to the value $\sigma$. When passing through the node $i=k$, the diagram undergoes a jump, which is greater, the smaller $\sigma$. Regardless of $i_{0}=k-C_{0} / 2$, a constant layer $C_{0}$, is always set on the roller $C_{0}$, i.e. all distribution curves merge here. The layer $C_{0}$ becomes larger in the presence of an offset cylinder and with a decrease in the coefficient of transition of ink to paper $\beta_{p}$.

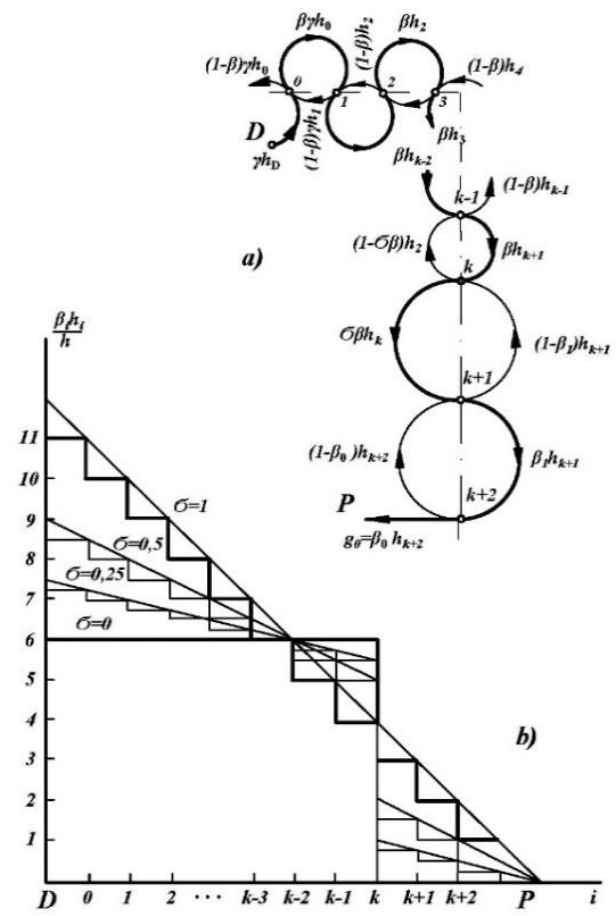

Figure 2. The distribution of layers of ink in the inking apparatus: a- motion scheme; b - distribution diagram in the direction of direct flow

Using the formula (9), the regularity of the ink distribution over the maximum layers in the direction of the direct flow, and the minimum layers in the direction of the reverse flow $\left(1-\beta_{i}\right) h_{i} / h$

$h$ are established. Also, for each roller, the middle layer characterizing the amount of ink accumulated on the roller can be calculated. For simplicity of calculation, it is assumed that the path sections of the forward and reverse flows are equal to each other: 


$$
\frac{\widetilde{h}_{l}}{h}=\frac{\beta_{i-1} h_{i-1}+\left(1-\beta_{i}\right) h_{i}}{2 h}
$$

Imagine the distribution of the middle layer $\tilde{h}_{1} / \mathrm{h}$ along the sections of the ink path $l_{i} / l_{0}\left(l_{0}-\right.$ the length of the print). The total amount of ink accumulated in the inking apparatus is determined by the amount characterizing the area under the distribution curve:

$$
N_{0}=\sum_{i=1}^{k+2} \frac{\widetilde{h}_{l}}{\mathrm{~h}} \frac{l_{i}}{l_{0}}=C_{0} \bar{d} k+\frac{1}{2}\left(2 i_{0}-k\right) \sigma \bar{d} k+\left(2+\frac{3}{\beta_{p}}\right) \sigma
$$

The first term of the sum characterizes the capacity of the rollers under the condition of uniform distribution $(\bar{d}$ is the ratio of the average diameter of the roller to the diameter of the plate cylinder); the second term takes into account the uneven distribution; the third is related to the capacity of the plate and offset cylinders.

The proposed method extended to inking devices with branched streams of ink (as shown in Figure 3).

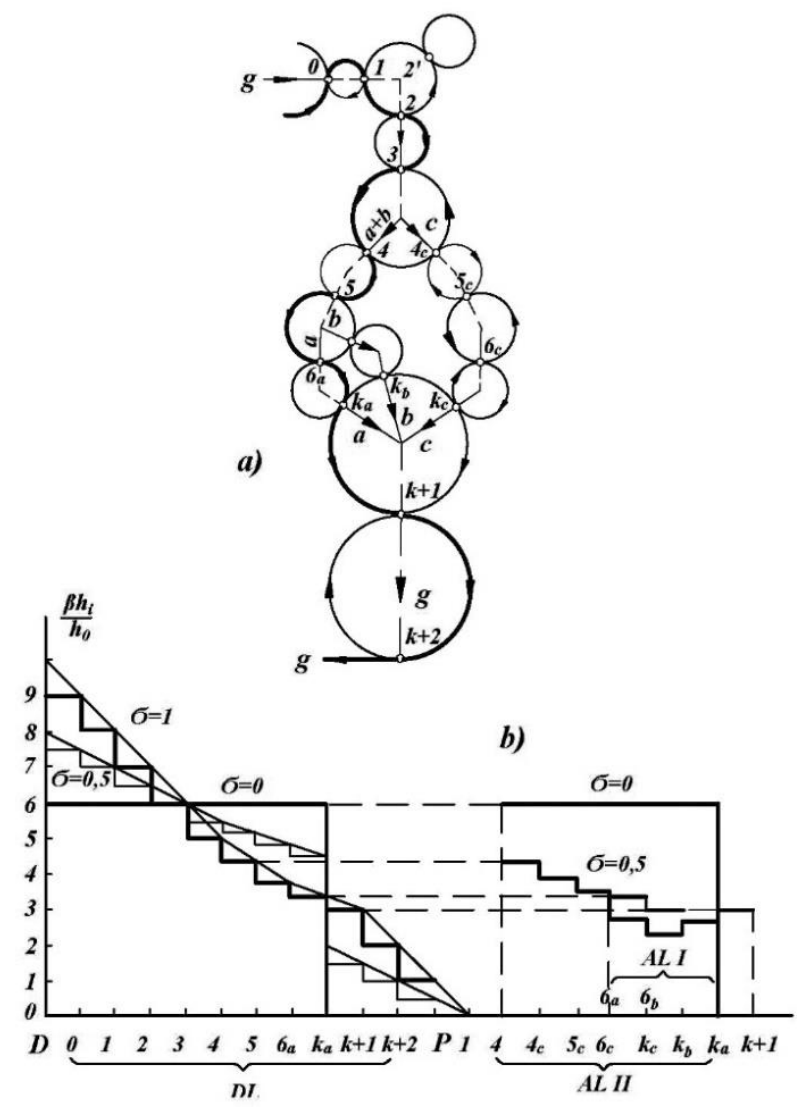

Figure 3. The distribution of ink in the inking apparatus of complex structure: a- motion scheme; b- distribution diagram; DL baseline AL I, AL II - additional lines

For this purpose, a scheme for branching ink flows passing through contact nodes is constructed (the sum of the components is equal to the total flow). A baseline is highlighted through which the ink enters the plate cylinder in the shortest possible way. For the baseline, the ink is distributed taking into account the attenuation of the ink flow due to branches. At the nodes where the ink flow branches, there are supply layers that serve as the beginning of additional lines. All additional lines end at node $k+1$. The distribution of layers in additional lines gives the necessary number of equations for determining the components of the total flow.

For non-stationary mode, the change in the volume of ink in the stream is characterized by the equation of the singlecapacitance model (the time is counted in printing cycles $n$ ).

$$
\frac{d V}{d n}=q_{t}-q_{f}
$$

where, $q_{\mathrm{t}}=h_{\mathrm{t}}$ the supply of ink to the site I x I, $q_{\mathrm{f}}=h$ - is the total consumption of ink from the site I x I taking into account the transfer of ink to the print elements of the print $q=\sigma h$ and returning the remaining amount of ink $q^{\prime}=(1-\sigma) h$ to the ink tank. In a similar equation by Mill [7], the return of ink $q^{\prime}$ was not taken into account. Given the amount of ink accumulated in the stream $V=N_{0} h$. Where $N_{0}$ - is the constant of the ink unit, we obtain the equation of the non-stationary mode.

$$
N_{0} \frac{d h}{d n}+h=h_{t}\left(n-n_{0}\right)
$$

where, $n_{0^{-}}$is the transport delay; the expression for the transient response takes the form.

$$
h(n)=h+\left(h_{t}+h_{0}\right)\left(1-e^{-\frac{n-n_{0}}{N_{0}}}\right)
$$

The constant $N_{0}$ is determined taking into account the actual distribution of inking the stream in accordance with the formula (18).

The dynamic characteristic (20) of the single-capacitance model allows one to determine the unevenness of the ink layer on the form in the case of pulsed power supply of the inking apparatus $h_{t}(t)$. Considering the inking apparatus as an open pulse system [15], using the discrete Laplace transform to solve Eq. (20), we find the amplitude of the pulsations with respect to the average layer thickness

$$
\Delta=\frac{h_{\max }-h_{\min }}{2 h_{\text {ave }}}=\frac{1-e^{-\frac{1-\gamma_{0}}{N_{0}}}}{1+e^{-\frac{1-\gamma_{0}}{N_{0}}}}
$$

Given an acceptable value $\Delta_{0}$, you can determine the required value of the constant $N_{0}$ of the inking apparatus.

\section{DISCUSSION}

As a result of the studies, the regularity of the distribution of the layers of ink in the inking apparatus was determined. To study the movement of ink, a single-capacitive model of an inking apparatus was developed. For this purpose, the flow of ink roughs a series of rollers and cylinders that form a baseline is considered (Figure 1a).

Modeling was carried out under certain conditions. The ratio of the formed layers was characterized by a splitting coefficient $B_{i}$ which are determined by formulas (2), (3), (4).

To determine the amount of ink $g_{i}$, passing through each contact node in the stationary mode, the power conditions of the ink apparatus are considered. The amount of ink $g_{0}$, supplied to the inking apparatus in the average for one printing 
cycle and the amount of ink transferred to the print is determined by equation (8). Based on this equation, taking into account the splitting coefficients, a mathematical model of the distribution of ink layers (formula 9) is constructed, and a method for calculating the distribution of ink layers in an inking apparatus of high (15) and offset (16) printing machines is also developed.

The regularity of the distribution of ink on the maximum and minimum layers is established. For each roller, the middle layer characterizing the amount of ink accumulated on the roller is calculated by formula (17). Based on theoretical studies, a diagram of the distribution of ink flow for the baseline is built (Figure 2). It was found that the $C_{0}$ layer becomes larger in the presence of an offset cylinder and with a decrease in the ink-to-paper transfer coefficient $\beta_{p}$.

The developed methodology for calculating the distribution of ink layers in an inking apparatus of high and offset printing machines can be extended to inking devices with branched streams of ink. To calculate the distribution of ink layers in this inking device, it is necessary to determine the pattern of branching of the ink flows passing through the contact nodes. Select the baseline through which the ink enters the mold cylinder in the shortest way. The supply layers located in the nodes, the branching of the ink flow is considered the beginning of additional lines. Based on the fact that all additional lines end at the node $k+1$, the distribution of layers in additional lines gives the necessary number of equations to determine the components of the total flow.

It is established that in an unsteady mode, a change in the volume of ink in the stream is also characterized by the equation of a single-capacitance model. The ink volume is determined by formula (19), taking into account the transfer of ink to the printing elements and returning the remaining amount of ink to the ink tank. In a similar equation, the return of ink was not taken into account, which led to erroneous results.

Studies have also established that the unevenness of the ink layer on the form in the case of pulsed power of the inking apparatus can be determined by the dynamic characteristic of the single-capacitance model, which is determined by formula (20).

The advantages of this study compared to analogs can be considered that, in contrast to analogs, a fundamentally different approach to the mathematical description of inking devices based on the method of structural and mathematical modeling is proposed. The inking apparatus was considered as a dynamic system formed by a combination of rollers and cylinders and converting the input action (continuous or discrete portions of ink) into a time-varying thickness of the intermediate and output layers.

The mathematical description is based on a system of difference or differential equations that adequately reflect the structure of the inking apparatus and the dynamic processes that occur in its links. The transitional characteristic of the inking apparatus was considered as a natural reaction of this model to various external influences (ink supply, shape relief, etc.).

\section{CONCLUSIONS}

1. It has been established that a single-capacitance model represents an inking apparatus as a single continuous flow, characterized by a change in the layer thickness along the line of ink movement, as well as in time.

2. It was found that the pulsed power supply at the input of this model makes it possible to determine the unevenness of the ink layer on the form

3. The calculations established that in stationary mode the amount of ink $g_{i}$ passing through each contact node for one printing cycle is constant. The amount of ink $g_{i}$ is equal to the amount of ink $g_{0}$ supplied to the inking apparatus on average for one printing cycle and the amount of ink transferred to the print.

4. The proposed methodology for calculating the distribution of ink layers can be extended to inking devices with branched streams of ink.

5. The change in the volume of ink in the stream in an unsteady mode and the unevenness of the ink layer on the form in the case of pulsed power of the inking apparatus is characterized by the equation of a single-capacitance model.

\section{REFERENCES}

[1] Bobrov, V.I., Kulikov, G.B. (2012). Problems of creating and using effective printing and finishing systems. Proceedings of Universities, Problems of Printing and Publishing, 2: 12-14.

[2] Wilson, D.J. (2007). Web offset printing machine: mechanisms, operation, maintenance. ed. V.N. Rumyantsev. M.: Print-Media Center. https://books.google.az/books/about/.html?id= yDfNw AACAAJ\&redir_esc $=y$.

[3] Lee, A.R., Kim, I., Kim, K.Y., Nam, S.Y., Choi, Y.M. (2015). Quantitative measurement of ink-blanket adhesion for contact transfer printing inks. International Journal of Precision Engineering and Manufacturing. 16(1): 151-156. https://doi.org/10.1007/s12541-0150019-z

[4] Su, L., Chu, H.Y., Cai, L.G., Zhao, J.T. (2012). Research of elastic deformation of rubber ink roller and ink flow characteristics on two ink rollers. Applied Mechanics and Materials, 220-223: 1703-1710. https://doi.org/10.4028/www.scientific.net/AMM.220223.1703

[5] Verkhola, M.I., Panovik, U.P. (2015). Information technology for the analysis of ink transfer in ink printing systems of offset printing machines. Transactions of BSTU. Publishing and Printing, 9(182): 18-23.

[6] Morozov, M.G. (1968). Calculation of ink supply in letterpress and flat printing machines. part I, MSH, 19: 84-102.

[7] Mill, K.K. (1964). Experimental verification of the theory of ink distribution. In the Book: Printing Inks and Color, 366-385.

[8] Ruder, R. (1965). Unter suchungenzur Farbverteilungim Farbwerken von Rotationsmaechinen des Hoch-und Flachdruckes. T.H. Karl $\neg$ Marx-Stadt, 32-34.

[9] Alekseyev, G.A. (1980). Inking machines of rotational letterpress and flat printing machines. M.: Book, 74-82.

[10] Aliyev, E.A. (2017). Influence microgeometry offset printing plates for transfer ink from the printing form on Dekel. Proceedings of the International Symposium of Mechanism and Machine Science, AzCIFToMMAzerbaijan Technikal University, Baku, Azerbaijan, pp. 201-202.

[11] Liu, L.L., Li, K.K., Lu, F. (2016). Dynamic simulation 
modeling of inking system based on elastohydrodynamic lubrication, International Journal of Heat and Technology, 34(1): 124-128. https://doi.org/10.18280/ijht.340118

[12] Liu, L.L., Lu, F., Bai, J.Y. (2011). Simulation of inking system based on elastohydrodynamic lubrication. 2nd International Conference on Frontiers of Manufacturing and Design Science (ICFMD 2011). Applied Mechanics and Materials, 121-126: 2883-2886. https://doi.org/10.4028/www.scientific.net/AMM.121126.2883

[13] Zhao, M.L. (2011). The dynamic property analysis of ink system in offset press. 2nd International Conference on Manufacturing Science and Engineering. Advanced Materials Research, 199-200: 132-136. https://doi.org/10.4028/www.scientific.net/AMR

[14] Yan, Z.H., Hui, R.L., Ling, H.Y. (2009). Dynamic simulation of offset printing inking system based on undirected graph. ETP / IITA World Congress in Applied Computing, Computer Science and Computer Engineering (ACC 2009), Sanya, Peoples R. China, pp. 193-196.

[15] Dittkin, Y.Z. (1963). Theory of linear impulse systems. M.: Fizmatiz, 164-176. 